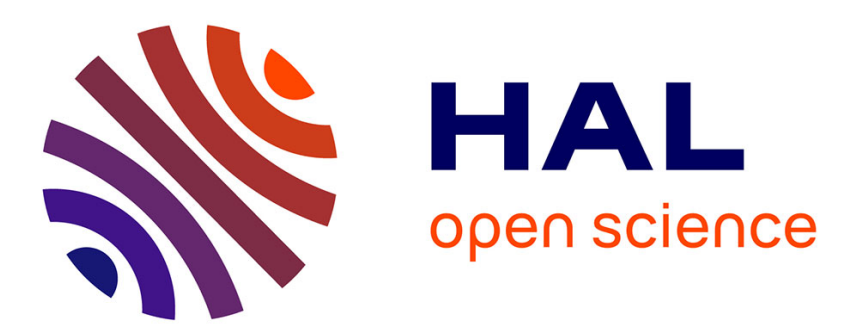

\title{
Migration of an insulating particle under the action of uniform ambient electric and magnetic fields. Part 1. General theory
}

\author{
H.K. Moffatt, Antoine Sellier
}

\section{- To cite this version:}

H.K. Moffatt, Antoine Sellier. Migration of an insulating particle under the action of uniform ambient electric and magnetic fields. Part 1. General theory. Journal of Fluid Mechanics, 2002, 464 (august), pp.279-286. 10.1017/s0022112002001131 . hal-01024913

HAL Id: hal-01024913

https://hal-polytechnique.archives-ouvertes.fr/hal-01024913

Submitted on 3 Sep 2014

HAL is a multi-disciplinary open access archive for the deposit and dissemination of scientific research documents, whether they are published or not. The documents may come from teaching and research institutions in France or abroad, or from public or private research centers.
L'archive ouverte pluridisciplinaire HAL, est destinée au dépôt et à la diffusion de documents scientifiques de niveau recherche, publiés ou non, émanant des établissements d'enseignement et de recherche français ou étrangers, des laboratoires publics ou privés. 


\title{
Migration of an insulating particle under the action of uniform ambient electric and magnetic fields. Part 1. General theory
}

\author{
By H. K. MOFFATT ${ }^{1} \dagger$ AND A. SELLIER ${ }^{2}$ \\ ${ }^{1}$ Laboratoire de Physique Statistique, Ecole Normale Supérieure, Rue Lhomond, \\ 75231 Paris, France \\ ${ }^{2}$ LadHyX, Ecole Polytechnique, 91128 Palaiseau, France
}

(Received 3 April 2002 and in revised form 30 April 2002)

The behaviour of an insulating particle suspended in a liquid metal and subject to the influence of locally uniform electric and magnetic fields $(\boldsymbol{E}, \boldsymbol{B})$ is considered. The electric field drives a current $\boldsymbol{J}$ which is perturbed by the presence of the particle, and the resulting Lorentz force drives a flow. It is assumed that both the Reynolds number and the Hartmann number based on particle size are small. If the particle is fixed, it experiences a force and couple that are each bilinear in $\boldsymbol{J}$ and $\boldsymbol{B}$; if it is freely suspended, then it moves with translational velocity $\boldsymbol{U}$ and angular velocity $\boldsymbol{\Omega}$ each similarly bilinear in $\boldsymbol{J}$ and $\boldsymbol{B}$. The general form of these bilinear relationships is determined, with particular attention to three types of particle symmetry: (i) isotropy; (ii) axisymmetry; and (iii) orthotropy.

\section{Introduction}

It has been shown both theoretically (Leenov \& Kolin 1954) and experimentally (Marty \& Alemany 1984) that a particle immersed in a liquid metal of different conductivity may be forced to migrate relative to the liquid through the combined influence of applied electric and magnetic fields. The current that is driven by the electric field interacts with the magnetic field to produce a rotational Lorentz force. This drives a flow which exerts a viscous stress on the particle causing it to move relative to the remote fluid. The technique may be used to accelerate the sedimentation of impurities in liquid metals, and therefore has an important practical application.

The previous studies were restricted to particles of idealized form, either spherical or cylindrical. In practice, particle impurities may be of very irregular shape, and it is desirable to construct a theory for particles of arbitrary shape. This is the purpose of the present paper, which is based on the assumption that the particle is small enough for all inertial effects to be negligible, and for the Hartmann number based on particle size to be small. In these circumstances, there is a decoupling of the electromagnetic problem and the dynamic problem, and both problems are linear. This allows considerable progress using just principles of linearity and symmetry.

$\dagger$ On leave from Department of Applied Mathematics and Theoretical Physics, University of Cambridge, Silver Street, Cambridge, UK. 


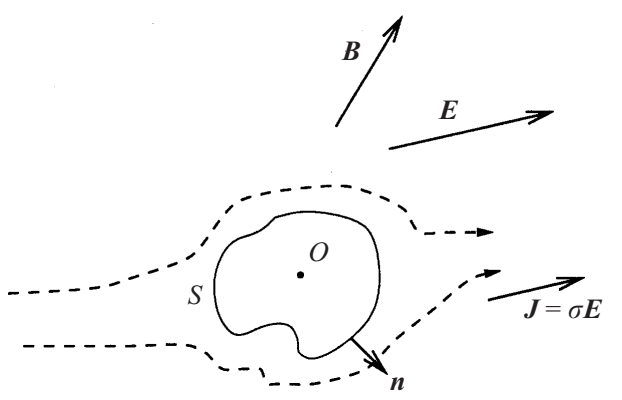

FIGURE 1. A solid particle subject to ambient electric and magnetic fields $\boldsymbol{E}$ and $\boldsymbol{B}$. The dashed lines are the perturbed current lines.

\section{Electromagnetic force $\boldsymbol{F}^{E}$ and couple $G^{E}$ acting on a fixed particle}

Consider first the problem of a particle that is held fixed at some interior point of a liquid metal of uniform density $\rho$, viscosity $\mu$, and conductivity $\sigma$, where the ambient electric field $\boldsymbol{E}$ and magnetic field $\boldsymbol{B}$ take the locally uniform values $\left(\boldsymbol{E}^{\infty}, \boldsymbol{B}^{\infty}\right)$, the superfix ' $\infty$ ' simply indicating that these are the fields 'far from the particle', or equivalently the fields that would be present if the particle were not there.

The field $\boldsymbol{E}$ drives a current $\boldsymbol{J}=\sigma \boldsymbol{E}$ and the resulting Lorentz force density is $\boldsymbol{J} \wedge \boldsymbol{B}$. We suppose that this force is compensated by a pressure gradient, i.e.

$$
\boldsymbol{J} \wedge \boldsymbol{B}=\nabla p,
$$

where $p(\boldsymbol{x})$ is a pressure field sustained by the pressure distribution on distant fluid boundaries (just as the gravitational force is compensated by a hydrostatic pressure gradient). The current $\boldsymbol{J}$ itself contributes to the magnetic field $\boldsymbol{B}$ via Ampère's Law; but in practical circumstances, the dominant contribution to $\boldsymbol{B}$ comes from electromagnets external to the fluid domain. The magnetostatic condition (2.1) is here adopted merely for simplicity of exposition; if it is not satisfied, then there is a bulk motion of the liquid metal which can be taken into account without difficulty.

We suppose that the particle has surface $S$, centre of volume $O$, and length scale $a$ (which could for example be the radius of a sphere of volume $V$ equal to that of the particle). We suppose that $a$ is sufficiently small compared with the scale of the fields $\boldsymbol{E}(\boldsymbol{x}), \boldsymbol{B}(\boldsymbol{x})$ for the locally uniform approximation to be indeed valid. We may then safely drop the superfix $\infty$ on the fields $\boldsymbol{E}^{\infty}, \boldsymbol{J}^{\infty}, \boldsymbol{B}^{\infty}$, as we do from now on for simplicity of notation. The current $\boldsymbol{J}$ is perturbed to $\boldsymbol{j}(\boldsymbol{x})$, say, by the presence of the particle, if its conductivity $\sigma_{p}$ differs from that of the fluid. We shall assume for simplicity that the particle is insulating $\left(\sigma_{p}=0\right)$. Then $\boldsymbol{j}(\boldsymbol{x})$ satisfies the condition $\boldsymbol{j} \cdot \boldsymbol{n}=0$ on $S$, where $\boldsymbol{n}$ is the unit outward normal on $S$, and $\boldsymbol{j}$ tends to $\boldsymbol{J}$ far from the particle. The situation is sketched in figure 1. (It is straightforward to extend the results to the case of conducting particles $\sigma_{p}>0$; this simply involves solving for $\boldsymbol{j}(\boldsymbol{x})$ inside, as well as outside, the particle, using continuity of the normal component of $\boldsymbol{j}(\boldsymbol{x})$ and the tangential component of $\boldsymbol{E}(\boldsymbol{x})$ across the particle surface.)

The Lorentz force density $\boldsymbol{f}(\boldsymbol{x})=\boldsymbol{j} \wedge \boldsymbol{B}$ is in general rotational, and drives a steady flow $\boldsymbol{u}(\boldsymbol{x})$ in the neighbourhood of the particle, of scale $U$, say. We assume that the Reynolds number $R e=\rho U a / \mu$ is small, and that all inertial effects are therefore negligible. The velocity $\boldsymbol{u}$ is then determined by the Stokes equations

$$
\mu \nabla^{2} \boldsymbol{u}=\nabla p-\boldsymbol{f}, \quad \nabla \cdot \boldsymbol{u}=0,
$$


with boundary conditions

$$
\boldsymbol{u}_{S}=\mathbf{0}, \boldsymbol{u}_{\infty}=\mathbf{0}, \quad p \sim(\boldsymbol{J} \wedge \boldsymbol{B}) \cdot \boldsymbol{x} \quad \text { at } \infty,
$$

where $\boldsymbol{u}_{S}$ is the value of $\boldsymbol{u}$ on $S$, and $\boldsymbol{u}_{\infty}$ is the value of $\boldsymbol{u}$ 'at infinity'. Clearly, the order of magnitude of $\boldsymbol{u}$ is given by

$$
U=J B a^{2} / \mu,
$$

where $J=|\boldsymbol{J}|, B=|\boldsymbol{B}|$, and the condition $R e \ll 1$ becomes

$$
R e=\rho J B a^{3} / \mu^{2} \ll 1 .
$$

This criterion is satisfied in practice for particles of scales of order $1 \mathrm{~mm}$ and less, a range that is of relevance in the purification context. The flow $\boldsymbol{u}(\boldsymbol{x})$ has components across $\boldsymbol{B}$ and therefore induces an additional current of order $\sigma U B$. This is negligible compared with $J$ provided

$$
M^{2}=\sigma B^{2} a^{2} / \mu \ll 1 .
$$

$M$ is the Hartmann number, invariably small for particles of the scale of $1 \mathrm{~mm}$ or less. Thus this induced current may be neglected. Note that the perturbation magnetic field associated with the induced current has order-of-magnitude $\operatorname{Re}_{m} B$ where, with $\mu_{0}$ the standard electromagnetic permeability, $R e_{m}=\mu_{0} \sigma U a$ is the magnetic Reynolds number, invariably much smaller than $R e$ in the liquid metal context (Moreau 1990). Thus, it is indeed legitimate to assume that $\boldsymbol{B}$ is uniform.

It follows from the above considerations that the current distribution may be calculated as if the fluid were at rest. This electrostatic problem is thus decoupled from the dynamical problem of determining $\boldsymbol{u}(\boldsymbol{x})$, a very considerable simplification. Writing $\boldsymbol{j}=-\sigma \nabla \varphi$, the electrostatic potential $\varphi$ is uniquely determined (up to a cnstant) by solution of the exterior Neumann problem

$$
\begin{gathered}
\nabla^{2} \varphi=0, \\
\partial \varphi / \partial n=0 \quad \text { on } S, \quad-\sigma \nabla \varphi \sim \boldsymbol{J} \quad \text { at } \infty .
\end{gathered}
$$

Clearly $\sigma \varphi$, and so $\boldsymbol{j}$, are linearly related to $\boldsymbol{J}$. Hence the Lorentz force density $\boldsymbol{f}(\boldsymbol{x})=\boldsymbol{j} \wedge \boldsymbol{B}$ is bilinear in $\boldsymbol{J}$ and $\boldsymbol{B}$.

Returning now to the dynamical problem (2.2), (2.3), it is evident that $\boldsymbol{u}(\boldsymbol{x})$ is linearly related to $\boldsymbol{f}(\boldsymbol{x})$ and is therefore also bilinear in $\boldsymbol{J}$ and $\boldsymbol{B}$. We shall use suffix notation and standard summation convention throughout. Let $\sigma_{i j}$ be the Cartesian components of the associated stress tensor, defined by

$$
\sigma_{i j}=-p \delta_{i j}+\mu\left(\partial u_{i} / \partial x_{j}+\partial u_{j} / \partial x_{i}\right) .
$$

Then the force $\boldsymbol{F}^{E}$ and couple $\boldsymbol{G}^{E}$ (both of electromagnetic origin) acting on the particle are given by

$$
F_{i}^{E}=\int_{S} \sigma_{i j} n_{j} \mathrm{~d} S, \quad G_{i}^{E}=\epsilon_{i j k} \int_{S} x_{j} \sigma_{k l} n_{l} \mathrm{~d} S,
$$

where $\boldsymbol{x}$ is measured from the centre of volume $O$. The linearity of (2.9) and (2.10) now implies that $\boldsymbol{F}^{E}$ and $\boldsymbol{G}^{E}$ are, like $\boldsymbol{u}(\boldsymbol{x})$, bilinear in $\boldsymbol{J}$ and $\boldsymbol{B}$.

Now $\boldsymbol{J}$ is a pure vector, whereas $\boldsymbol{B}$ is a pseudo- (or 'axial') vector. Moreover, $\boldsymbol{F}^{E}$ has the dimensions of $a^{3} J B$. Hence we may express the bilinear dependence of $\boldsymbol{F}^{E}$ on $\boldsymbol{J}$ and $\boldsymbol{B}$ in the form

$$
F_{i}^{E}=a^{3} A_{i j k} J_{j} B_{k},
$$


where $A_{i j k}$ is a dimensionless third-rank pseudo-tensor which is determined (in principle) solely by the shape of the particle; it is natural to describe $A_{i j k}$ as a "particle pseudo-tensor', since it is a property intrinsic to the particle. (A pseudo-tensor is distinguished from a tensor by its transformation properties under a 'parity transformation' from a right-handed to a left-handed frame of reference: the determinant of such a transformation, equal to -1 , is included in the transformation law for a pseudo-tensor, but not for a tensor. Thus, for example, a pseudo-scalar like $\boldsymbol{J} \cdot \boldsymbol{B}$ changes sign under parity transformations.) Similarly,

$$
G_{i}^{E}=a^{4} C_{i j k} J_{j} B_{k},
$$

where, since $\boldsymbol{G}^{E}$ is a pseudo-vector, $C_{i j k}$ is a dimensionless third-rank tensor likewise determined solely by the shape of the particle (i.e. a 'particle tensor').

We may note here that, for the case of a particle of non-zero conductivity $\sigma_{p}$, all the above arguments go through with trivial modification, the only consequence being that $A_{i j k}, C_{i j k}$ become functions of the conductivity ratio $\kappa=\sigma_{p} / \sigma>0$, vanishing when $\kappa=1$ (since then $\boldsymbol{j}=\boldsymbol{J}$ and the fluid remains at rest).

\section{Symmetry considerations}

Symmetry considerations can place strong constraints on the form of $A_{i j k}$ and $C_{i j k}$, as shown by the following examples.

\subsection{Isotropic particles}

We shall describe as 'isotropic' any particle for which all particle tensors or pseudotensors such as $C_{i j k}$ and $A_{i j k}$ are isotropic. The sphere is of course the prototype; but it is by no means the only such particle. It is sufficient that the particle have at least three planes of symmetry whose normals are linearly independent. This condition is satisfied by all the regular solids (tetrahedron, cube, octahedron, dodecahedron or icosahedron) and any particle having the symmetry of any regular solid is, from this point of view, isotropic. For any such particle, $A_{i j k}$ is isotropic, i.e. (being a pseudo-tensor)

$$
A_{i j k}=\alpha \epsilon_{i j k}, \quad \text { and so } \quad \boldsymbol{F}^{E}=\alpha a^{3} \boldsymbol{J} \wedge \boldsymbol{B},
$$

where $\alpha$ is a number solely determined by the shape of the particle. Thus, $\boldsymbol{F}^{E}$ is aligned with (i.e. parallel or anti-parallel to) $\boldsymbol{J} \wedge \boldsymbol{B}$, and independent of particle orientation. This result is known for the sphere, for which $\alpha=-\pi$ (Leenov \& Kolin 1954), but the present argument shows it to be equally true for any regular solid, or for any particle of equivalent symmetry.

The tensor $C_{i j k}$, being also isotropic, must vanish, and so $\boldsymbol{G}^{E}=\mathbf{0}$. Note however that if reflectional symmetry is relaxed, then we may have

$$
C_{i j k}=\gamma \epsilon_{i j k}, \quad \text { and so } \quad \boldsymbol{G}^{E}=\gamma a^{4} \boldsymbol{J} \wedge \boldsymbol{B},
$$

where $\gamma$ is a pseudo-scalar reflecting the handedness, or 'chirality', of the particle. For example, an amalgam of twelve right-handed helices with their axes aligned on the edges of a cube and fused together at the eight corners would give $\gamma \neq 0$. The mirror image of this (admittedly exotic) particle, having left-handed helices, would have $C_{i j k}=-\gamma \epsilon_{i j k}$.

\subsection{Axisymmetric particles}

Consider now a particle having an axis of symmetry aligned with the unit vector $\boldsymbol{e}$, say. The pseudo-tensor $A_{i j k}$ is then an axisymmetric pseudo-tensor function of $\boldsymbol{e}$, and 

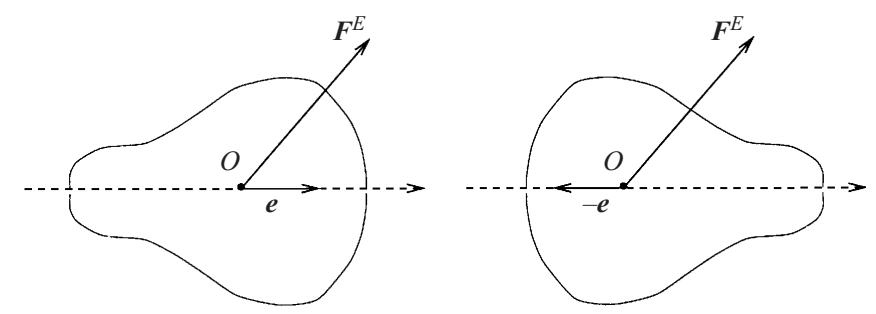

FIGURE 2. A pear-shaped particle with an axis of symmetry parallel to $\boldsymbol{e}$. The electromagnetic force $\boldsymbol{F}^{E}$ is invariant under the flip, as indicated.

necessarily takes the form

$$
A_{i j k}=\alpha^{(0)} \epsilon_{i j k}+\alpha^{(1)} \epsilon_{p j k} e_{p} e_{i}+\alpha^{(2)} \epsilon_{i p k} e_{p} e_{j}+\alpha^{(3)} \epsilon_{i j p} e_{p} e_{k},
$$

where the coefficients $\alpha^{(0)}, \ldots, \alpha^{(3)}$ are scalars determined by the shape of the particle. The corresponding expression for $\boldsymbol{F}^{E}$ is

$$
\boldsymbol{F}^{E}=a^{3}\left\{\alpha^{(0)} \boldsymbol{J} \wedge \boldsymbol{B}+\alpha^{(1)}[\boldsymbol{e} \cdot(\boldsymbol{J} \wedge \boldsymbol{B})] \boldsymbol{e}+\alpha^{(2)}(\boldsymbol{e} \cdot \boldsymbol{J})(\boldsymbol{e} \wedge \boldsymbol{B})+\alpha^{(3)}(\boldsymbol{e} \cdot \boldsymbol{B})(\boldsymbol{J} \wedge \boldsymbol{e})\right\} .
$$

This force is generally non-zero even when $\boldsymbol{J}$ is parallel to $\boldsymbol{B}$ ! For then, (3.4) gives

$$
\boldsymbol{F}^{E}=a^{3}\left(\alpha^{(2)}-\alpha^{(3)}\right)(\boldsymbol{e} \cdot \boldsymbol{B})(\boldsymbol{e} \wedge \boldsymbol{J}),
$$

and this is in general non-zero provided $\boldsymbol{e}$ is neither parallel nor perpendicular to $\boldsymbol{B}$.

Suppose now that $\boldsymbol{J} \wedge \boldsymbol{B}$ is non-zero. It is evident from (3.4) that in general $\boldsymbol{F}^{E}$ is not aligned with $\boldsymbol{J} \wedge \boldsymbol{B}$. However, there are two circumstances for which $\boldsymbol{F}^{E}$ is aligned with $\boldsymbol{J} \wedge \boldsymbol{B}$ :

(i) if $\boldsymbol{e}$ is parallel to $\boldsymbol{J} \wedge \boldsymbol{B}$, then obviously

$$
\boldsymbol{F}^{E}=a^{3}\left(\alpha^{(0)}+\alpha^{(1)}\right) \boldsymbol{J} \wedge \boldsymbol{B}
$$

(ii) if $\boldsymbol{e}$ lies in the plane normal to $\boldsymbol{J} \wedge \boldsymbol{B}$, so that $\boldsymbol{e}=p \boldsymbol{J}+q \boldsymbol{B}$, where

$$
p=\frac{\left\{B^{2}(\boldsymbol{e} \cdot \boldsymbol{J})-(\boldsymbol{e} \cdot \boldsymbol{B})(\boldsymbol{J} \cdot \boldsymbol{B})\right\}}{(\boldsymbol{J} \wedge \boldsymbol{B})^{2}}, \quad q=\frac{\left\{\boldsymbol{J}^{2}(\boldsymbol{e} \cdot \boldsymbol{B})-(\boldsymbol{e} \cdot \boldsymbol{J})(\boldsymbol{J} \cdot \boldsymbol{B})\right\}}{(\boldsymbol{J} \wedge \boldsymbol{B})^{2}},
$$

then

$$
\boldsymbol{F}^{E}=a^{3}\left\{\alpha^{(0)}+\alpha^{(2)} p(\boldsymbol{e} \cdot \boldsymbol{J})+\alpha^{(3)} q(\boldsymbol{e} \cdot \boldsymbol{B})\right\} \boldsymbol{J} \wedge \boldsymbol{B} .
$$

Note the particular cases $\boldsymbol{e}=\boldsymbol{B} / B$ (when $p=0$ ) and $\boldsymbol{e}=\boldsymbol{J} / J$ (when $q=0$ ). Four independent measurements of $\boldsymbol{F}^{E}$ for different orientations of $\boldsymbol{e}$ will clearly suffice to determine $\alpha^{(0)}, \ldots, \alpha^{(3)}$. Note from (3.4) that $\boldsymbol{F}^{E}$ is invariant under replacement of $\boldsymbol{e}$ by $-\boldsymbol{e}$, i.e. the force is unchanged by flipping the axis of the particle through an angle $\pi$. This is of course obvious for a 'flip-invariant' particle, i.e. one which is symmetric about the plane through $O$ normal to $e$, but it is far from obvious for particles that lack this 'flip-symmetry' (e.g. the pear-shaped particle shown in figure 2). The (pure) tensor $C_{i j k}$ is similarly an axisymmetric tensor function of $\boldsymbol{e}$, and therefore takes the form

$$
C_{i j k}=\gamma^{(0)} e_{i} e_{j} e_{k}+\gamma^{(1)} e_{i} \delta_{j k}+\gamma^{(2)} e_{j} \delta_{k i}+\gamma^{(3)} e_{k} \delta_{i j}
$$

where again the scalars $\gamma^{(0)}, \ldots, \gamma^{(3)}$ are determined solely by particle geometry. The corresponding couple is

$$
\boldsymbol{G}^{E}=a^{4}\left[\gamma^{(0)}(\boldsymbol{e} \cdot \boldsymbol{J})(\boldsymbol{e} \cdot \boldsymbol{B}) \boldsymbol{e}+\gamma^{(1)}(\boldsymbol{J} \cdot \boldsymbol{B}) \boldsymbol{e}+\gamma^{(2)}(\boldsymbol{e} \cdot \boldsymbol{J}) \boldsymbol{B}+\gamma^{(3)}(\boldsymbol{e} \cdot \boldsymbol{B}) \boldsymbol{J}\right] .
$$

Note here that $\boldsymbol{G}^{E}$ changes sign under the flip $\boldsymbol{e} \rightarrow-\boldsymbol{e}$; hence $\boldsymbol{G}^{E}$ must be zero for 


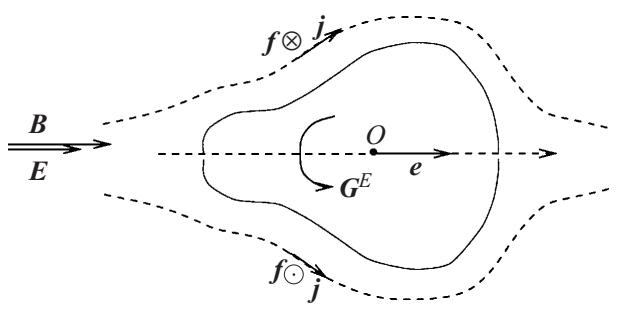

FIGURE 3. For a particle that is not flip symmetric, the net couple $\boldsymbol{G}^{E}$ relative to the centre of volume $O$ is non-zero even when both $\boldsymbol{E}$ and $\boldsymbol{B}$ are aligned with $\boldsymbol{e}$.

flip-invariant particles. However, for particles lacking this flip symmetry, $\boldsymbol{G}^{E}$ is in general non-zero. Surprisingly, this is the case even if both $\boldsymbol{J}$ and $\boldsymbol{B}$ are aligned with $\boldsymbol{e}$; for then, from (3.10),

$$
\boldsymbol{G}^{E}=a^{4}\left(\gamma^{(0)}+\gamma^{(1)}+\gamma^{(2)}+\gamma^{(3)}\right)(\boldsymbol{J} \cdot \boldsymbol{B}) \boldsymbol{e} .
$$

The origin of this couple may be understood with reference to figure 3: the Lorentz force $\boldsymbol{j} \wedge \boldsymbol{B}$ exerts a torque on the fluid which is not antisymmetric about the plane through $O$ normal to $\boldsymbol{e}$; the net torque transmitted to the particle by the viscous stress generated is then in general non-zero.

Finally, observe that $\boldsymbol{G}^{E}$ is aligned with $\boldsymbol{e}$ not only when both $\boldsymbol{J}$ and $\boldsymbol{B}$ are parallel to $\boldsymbol{e}$ (see (3.11)) but also when (as in case (i) above) $\boldsymbol{e}$ is parallel to $\boldsymbol{J} \wedge \boldsymbol{B}$. In this case, both $\boldsymbol{F}^{E}$ and $\boldsymbol{G}^{E}$ are aligned with $\boldsymbol{e}$ and

$$
\boldsymbol{G}^{E}=a^{4} \gamma^{(1)}(\boldsymbol{J} \cdot \boldsymbol{B}) \boldsymbol{e}
$$

The non-zero value of the pseudo-scalar 'wrench' $\boldsymbol{F}^{E} \cdot \boldsymbol{G}^{E}$ here arises from the fact that the pseudo-scalar $\boldsymbol{J} \cdot \boldsymbol{B}$ associated with the applied fields is also non-zero.

\subsection{Orthotropic particles}

An orthotropic particle is one that has three orthogonal planes of symmetry through its centre of volume $O$. The prototype is the ellipsoid. The unit normals to the three planes of symmetry $\boldsymbol{e}^{(1)}, \boldsymbol{e}^{(2)}, \boldsymbol{e}^{(3)}$ constitute an orthonormal triad. The particle is invariant under reflection in each of these planes, and hence (cf. equation (3.3)) $A_{i j k}$ may be expressed in the form

$$
A_{i j k}=\alpha^{(0)} \epsilon_{i j k}+\sum_{v=1}^{3}\left[\alpha^{(1 v)} \epsilon_{p j k} e_{p}^{(v)} e_{i}^{(v)}+\alpha^{(2 v)} \epsilon_{i p k} e_{p}^{(v)} e_{j}^{(v)}+\alpha^{(3 v)} \epsilon_{i j p} e_{p}^{(v)} e_{k}^{(v)}\right] .
$$

It is natural to adopt the frame of reference for which

$$
\boldsymbol{e}^{(1)}=(1,0,0), \quad \boldsymbol{e}^{(2)}=(0,1,0), \quad \boldsymbol{e}^{(3)}=(0,0,1) .
$$

In this frame,

$$
A_{i j k}=0 \quad \text { if any two of the indices } i, j, k \text { are equal. }
$$

From (2.11), we then have

$$
F_{1}^{E}=a^{3}\left(A_{123} J_{2} B_{3}+A_{132} J_{3} B_{2}\right),
$$

and similarly for $F_{2}^{E}, F_{3}^{E}$ by cyclic permutation of suffices. Note that $\boldsymbol{F}^{E}$ is aligned with $\boldsymbol{J} \wedge \boldsymbol{B}$ if the particle is oriented so that $\boldsymbol{J} \wedge \boldsymbol{B}$ is normal to any one of the planes of symmetry. 
The tensor $C_{i j k}$ can involve only products like $e_{i}^{(1)} \delta_{j k}$ or $e_{i}^{(1)} e_{j}^{(2)} e_{k}^{(3)}$ which change sign under appropriate reflections. Since $G^{E}$ is invariant under reflections, it follows that $C_{i j k}$ must in fact vanish. The couple $\boldsymbol{G}^{E}$ on an orthotropic particle is therefore always zero.

\section{Motion of a freely suspended particle}

Suppose now that the particle is freely suspended in the fluid; being without inertia, it then moves in such a way that the net force $\boldsymbol{F}$ and couple $\boldsymbol{G}$ acting upon it vanish. Let $\boldsymbol{U}$ be the velocity of the centre of volume $O$, and $\Omega$ the angular velocity of the particle relative to $O$. We then need to solve the Stokes problem

$$
\mu \nabla^{2} \boldsymbol{u}=\nabla p-\boldsymbol{f}, \quad \nabla \cdot \boldsymbol{u}=0,
$$

where $\boldsymbol{f}=\boldsymbol{j} \wedge \boldsymbol{B}$ as before, with the new boundary conditions

$$
\boldsymbol{u}_{S}=\boldsymbol{U}+\boldsymbol{\Omega} \wedge \boldsymbol{x}, \quad \boldsymbol{u}_{\infty}=0, \quad p \sim(\boldsymbol{J} \wedge \boldsymbol{B}) \cdot \boldsymbol{x} \quad \text { at } \infty .
$$

The unknown $\boldsymbol{U}$ and $\Omega$ are now to be determined through the auxiliary conditions

$$
F_{i}=\int_{S} \sigma_{i j} n_{j} \mathrm{~d} S=0, \quad G_{i}=\epsilon_{i j k} \int_{S} x_{j} \sigma_{k l} n_{e} \mathrm{~d} S=0 .
$$

The linearity of (4.1)-(4.3) allows us to decompose $\boldsymbol{u}$ as the sum $\boldsymbol{u}^{(1)}+\boldsymbol{u}^{(2)}$, where $\boldsymbol{u}^{(1)}$ and $\boldsymbol{u}^{(2)}$ satisfy:

\section{PRoblem 1.}

$$
\begin{gathered}
\mu \nabla^{2} \boldsymbol{u}^{(1)}=\nabla p^{(1)}-\boldsymbol{f}, \quad \nabla \cdot \boldsymbol{u}^{(1)}=0, \\
\boldsymbol{u}_{S}^{(1)}=0, \quad \boldsymbol{u}_{\infty}^{(1)}=0, \quad p \sim(\boldsymbol{J} \wedge \boldsymbol{B}) \cdot \boldsymbol{x} \quad \text { at } \infty ;
\end{gathered}
$$

Problem 2.

$$
\begin{gathered}
\mu \nabla^{2} \boldsymbol{u}^{(2)}=\nabla p^{(2)}, \quad \nabla \cdot \boldsymbol{u}^{(2)}=0, \\
\boldsymbol{u}_{S}^{(2)}=\boldsymbol{U}+\Omega \wedge \boldsymbol{x}, \quad \boldsymbol{u}_{\infty}^{(2)}=0, \quad p_{\infty}^{(2)}=0 .
\end{gathered}
$$

Here, problem 1 is precisely the problem that has been considered in $\S 2$; the corresponding force and couple on the particle are therefore

$$
\boldsymbol{F}^{(1)}=\boldsymbol{F}^{E}, \quad \boldsymbol{G}^{(1)}=\boldsymbol{G}^{E} .
$$

Problem 2 is the standard Stokes problem (see, for example, Happel \& Brenner 1973) which yields a force $\boldsymbol{F}^{(2)}$ and couple $\boldsymbol{G}^{(2)}$ linearly related to $\boldsymbol{U}$ and $\boldsymbol{\Omega}$ :

$$
\begin{aligned}
& F_{i}^{(2)}=-\mu\left(a \hat{A}_{i j} U_{j}+a^{2} \hat{B}_{i j} \Omega_{j}\right), \\
& G_{i}^{(2)}=-\mu\left(a^{2} \hat{B}_{j i} U_{j}+a^{3} \hat{D}_{i j} \Omega_{j}\right),
\end{aligned}
$$

where $\hat{A}_{i j}, \hat{D}_{i j}$ are dimensionless symmetric positive-definite particle tensors, and $\hat{B}_{i j}$ is a dimensionless particle pseudo-tensor. The linear relations (4.9)-(4.10) may equivalently be expressed in the inverse form

$$
\begin{aligned}
U_{i} & =-\mu^{-1}\left(a^{-1} \tilde{A}_{i j} F_{j}^{(2)}+a^{-2} \tilde{B}_{i j} G_{i j}^{(2)}\right), \\
\Omega_{i} & =-\mu^{-1}\left(a^{-2} \tilde{B}_{j i} F_{j}^{(2)}+a^{-3} \tilde{D}_{i j} G_{j}^{(2)}\right) .
\end{aligned}
$$


The conditions (4.3) imply that $\boldsymbol{F}^{(1)}+\boldsymbol{F}^{(2)}=0$ and $\boldsymbol{G}^{(1)}+\boldsymbol{G}^{(2)}=0$. Hence, from (4.8) and (4.11)-(4.12), we then have

$$
\begin{aligned}
U_{i} & =\mu^{-1}\left(a^{-1} \tilde{A}_{i j} F_{j}^{E}+a^{-2} \tilde{B}_{i j} G_{j}^{E}\right), \\
\Omega_{i} & =\mu^{-1}\left(a^{-2} \tilde{B}_{j i} F_{j}^{E}+a^{-3} \tilde{D}_{i j} G_{j}^{E}\right),
\end{aligned}
$$

or, substituting from (2.11) and (2.12),

$$
U_{i}=\mu^{-1} a^{2} M_{i p q} J_{p} B_{q}, \quad \Omega_{i}=\mu^{-1} a N_{i p q} J_{p} B_{q},
$$

where

$$
M_{i p q}=\tilde{A}_{i j} A_{j p q}+\tilde{B}_{i j} C_{j p q}, \quad N_{i p q}=\tilde{B}_{j i} A_{j p q}+\tilde{D}_{i j} C_{j p q} .
$$

$M_{i p q}$ and $N_{i p q}$ are dimensionless third-rank particle pseudo-tensor and tensor, respectively.

Obviously, the symmetry considerations of $\S 3$ apply equally to determine possible forms for $\left(M_{i p q}, N_{i p q}\right)$, and need not be repeated here. It may be useful however merely to summarize the conclusions that may be drawn:

(i) An isotropic particle (in the sense of §3.1) will move without rotation, and with constant velocity aligned with $\boldsymbol{J} \wedge \boldsymbol{B}$.

(ii) An axisymmetric particle which is not flip-symmetric has in general non-zero angular velocity; its orientation relative to $\boldsymbol{J} \wedge \boldsymbol{B}$ therefore changes with time and its velocity will therefore also in general be time-dependent.

(iii) An orthotropic particle (an axisymmetric particle with flip symmetry being a special case) does not rotate, and moves with constant velocity which is not generally aligned with $\boldsymbol{J} \wedge \boldsymbol{B}$, but is so aligned if $\boldsymbol{J} \wedge \boldsymbol{B}$ is normal to one of the planes of symmetry of the particle.

In Part 2 of this work (Sellier 2002), a boundary formulation is developed in a form suitable for the determination of the rigid-body motion $(\boldsymbol{U}, \Omega)$ of an arbitrary particle, and the analytical solution for the particular benchmark problem of an ellipsoid is obtained. The results are entirely consistent with the above conclusions.

A preliminary study of this problem was carried out by one of us (H.K.M.) in earlier collaboration with C. Economou under EPSRC Grant number GR/K50306, and was the subject of a poster presentation at the 19th International Congress of Theoretical and Applied Mechanics (Kyoto 1996). Ms Economou vanished from Cambridge in 1997; despite every attempt to locate her, her current whereabouts are unfortunately unknown.

\section{REFERENCES}

Happel, J. \& Brenner, H. 1973 Low Reynolds Number Hydrodynamics. Martinus Nijhoff.

LeEnov, D. \& Kolin, A. 1954 Theory of electromagnetophoresis. I. Magnetohydrodynamic forces experienced by spherical and symmetrically oriented cylindrical particles. J. Chem. Phys. 22, 683-688.

Marty, P. \& Alemany, A. 1984 Theoretical and experimental aspects of electromagnetic separation. In Metallurgical Applications of Magnetohydrodynamics (ed. H. K. Moffatt \& M. R. E. Proctor), pp. 245-259. Metals Society.

Moreau, R. 1990 Magnetohydrodynamics. Kluwer.

SEllier, A. 2002 Migration of an insulating particle under the action of uniform ambient electric and magnetic fields. Part 2. Boundary formulation and ellipsoidal particles. J. Fluid Mech. (submitted). 